\title{
Characterization of $\beta$-endorphin-related peptides in the caudal medulla oblongata and hypothalamus of the prenatal, postnatal and adult rat
}

\author{
Paul E. Quinlan and Norman E. Alessi \\ Department of Psychiatry, University of Michigan School of Medicine, Ann Arbor, MI 48109-0290 (U.S.A.)
}

(Accepted 19 March 1991)

Key words: Endorphin; Hypothalamus; Caudal medulla oblongata; Post-translational processing; Development

\begin{abstract}
A comparison was made of $\beta$-endorphin (B-END) concentrations versus post-translation products during the perinatal period in the hypothalamus and the caudal medulla oblongata. The concentration of B-END-like immunoreactivity did not differ statistically between embryonic day 21 (E21) and postnatal day 1 (P1) in either area. There were significant differences in forms, with a shift from larger precursors at E21 to smaller peptides at P1, with the predominant form of B-END being the 31 residue form at E21 in both regions. B-END varied between the two regions at $\mathrm{P} 1$, the 27-26 residue predominant in the hypothalamus, and the 31 residue in the caudal medulla.
\end{abstract}

\section{INTRODUCTION}

Pro-opiomelanocortin (POMC) and its post-translational products, e.g. $\beta$-lipotropin (B-LPH) $\beta$-endorphin (B-END), adrenocorticotropin (ACTH), and $\alpha$-melanocyte-stimulating hormone ( $\alpha$-MSH) have been identified in perikarya of the arcuate nucleus of the hypothalamus, the nucleus tractus solitarii of the caudal medulla oblongata and the anterior and intermediate lobes of the pituitary in the rat during postnatal development and adulthood $^{1-3,8,14,15,17,18,23}$. The first appearance of POMC in hypothalamic neurons occurs on embryonic day 12 and in the perikarya of the nucleus tractus solitarii on embryonic day $17^{12,13}$. In the pituitary, POMC appears in the anterior lobe on embryonic day 15 and in the intermediate lobe on embryonic day $16^{12}$. Radioimmunoassay studies of the nucleus tractus solitarii have demonstrated B-END-like immunoreactivity increases steadily from postnatal day 1 to postnatal day 42 and becomes comparable to adult levels ${ }^{2}$. Post-translational processing of B-END has been examined during development only in the pituitary ${ }^{25,26}$. Opioid activity of B-END can be significantly reduced through C-terminal cleavage as well as acetylation ${ }^{7,20,29}$. Seizenger ${ }^{26}$ found that in the neurointermediate lobe of the newborn rat the predominant form of B-END was 31-residue peptide in the acetylated form similar to the adult. The intermediate lobe also demonstrated decreased proteolysis of the B-END C-terminal. The anterior lobe demonstrated processing in the newborn similar to the adult with a higher proportion of the B-END-immunoreactive material in the larger forms and less acetylation of the B-END peptides. Sato ${ }^{25}$ found that in the neurointermediate lobe of the neonate rat the rate of C-terminal shortening was less than that found at postnatal day 21 and the adult age rat.

In human studies, the level of B-END immunoreactivity has been measured in the brainstem and the cerebellum of victims of Sudden Infant Death Syndrome ${ }^{21}$, ${ }^{22}$. The B-END immunoreacticity was found to be higher in the brainstem of victims of Sudden Infant Death Syndrome than in the control population. The depressant effect of opioids on respiration is a classic response. Moss found with injection of B-END into the cerebral spinal fluid of lightly anesthetized dogs there was a period of hypoventilation followed by hypotension and bradycardia $^{19}$. Increased respiratory function with the infusion of an opioid antagonist, naloxone, has been demonstrated in newborn rabbits ${ }^{11}$. The question which develops from these studies is whether or not the B-END peptides formed in the POMC perikarya of the caudal medulla oblongata and the hypothalamus undergo posttranslational processing at the time of parturition altering the peptide's opioid activity and its effect on respiration.

In the following study, we determine if the levels of 
B-END in the caudal medulla oblongata and hypothalamus change in accordance with birth and how these levels compare with the adult. In addition to peptide levels, the molecular forms of B-END-immunoreactive peptides were examined to determine the changes in post-translational processing.

\section{MATERIALS AND METHODS}

\section{Tissue preparation}

The rats selected for the study were of the Sprague-Dawley variety (Charles Rivers, Portland, MI). Paired adult male and virgin female rats were maintained in a 12-h light and 12-h dark schedule with food and water ad libitum. The female rats were examined daily for fertilization by vaginal saline lavage. Females with sperm present in the lavage were placed in individual cages. The date of conception, embryonic day 0 , was based on the first appearance of sperm. The embryonic day 21 rats were excised in utero and placed in iced saline until the time of decapitation. The postnatal day 1 rats were maintained with their mother for one day after birth prior to being sacrificed. The adult males used for tissue sampling were not used to fertilize females and were maintained in separate cages. All embryonic day 21, postnatal day 1 and adult rats were sacrificed by decapitation. The hypothalamic tissue containing the arcuate nucleus was dissected as a block of tissue as described by Glowinski ${ }^{9}$. Anatomical margins for dissection of the nucleus tractus solitarii from within the medulla oblongata consisted of the obex as the rostral boundary and the medulla-cord junction as the caudal boundary. The dissected tissue was placed on dry ice and stored at $-80^{\circ} \mathrm{C}$ prior to preparation for assay. Tissue extraction was performed as previously described ${ }^{3}$.

\section{Radioimmunoassay of $\beta$-endorphin}

The B-END radioimmunoassay (RIA) procedure was performed as previously described ${ }^{6}$. The assay consisted of a 3-day disequilibrium procedure using $4 \%$ sheep-anti-rabbit globulin serum as the second antibody. The peptide used as the standard and for iodination was human B-END (1-31) (Cat. No. 8616, Peninsula Lab, Belmont, CA). The antiserum was donated by Dr. Huda Akil (Mental Health Research Institute, University of Michigan). The $\mathrm{IC}_{50}$ for the assay was $8.6 \pm 0.7 \mathrm{fmol}$ per tube and the sensitivity was $4.0 \pm 0.3 \mathrm{fmol}$ per tube. The interassay coefficient of variation was $7.5 \%$ and the intraassay coefficient of variation was $6.8 \%$.

The B-END antiserum is $100 \%$ cross-reactivity with its larger molecular weight precursors, POMC and $\beta$-lipotropin. The antiserum showed no cross-reactivity with $\gamma$-endorphin [B-END(1-16)], ACTH, $\alpha$-MSH, $\gamma$-MSH, Leu- or Met-enkephalin, dynorphin-related peptides or a number of non-opioid pituitary peptides. A complete characterization of the antiserum can be found in Cahill et al. ${ }^{6}$. The B-END-like immunoreactivity measured in the RIA was the sum total of B-END, $\beta$-lipotropin and POMC immunoreactivities.

\section{Individual timepoint measurements}

The measurements of B-END levels at embryonic day 21, postnatal day 1 and adult were expressed as the mean \pm the standard error of the mean for 5 animals. The protein concentration of tissue samples was determined by a Folin phenol reagent procedure as described by Lowry ${ }^{16}$.

\section{Chromatography}

A $1.5 \times 90 \mathrm{~cm}$ column was prepared with G-50 superfine sepharose gel (Cat. No. G50-50, Sigma Chemical Co., St. Louis, MO). The eluent buffer was $10 \%$ formic acid, $0.1 \%$ BSA, and $0.1 \%$ 2-mercaptoethanol. A 2-mg sample of bovine serum albumin and 50- $\mu 1$ sample of 2-mercaptoethanol were added to the chromatographic samples to detect the $V_{\mathrm{o}}$ and $V_{\mathrm{t}}$, respectively, by means of ultraviolet spectrophotometry. The column flow rate was $5 \mathrm{ml}$ per

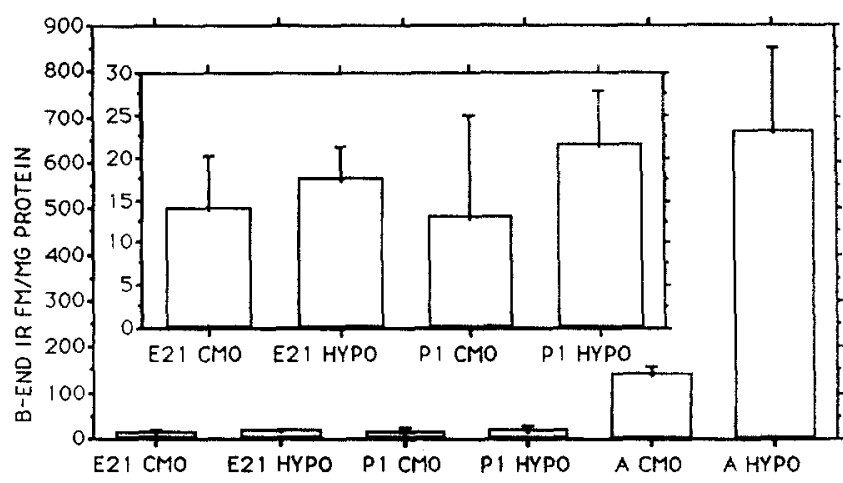

Fig. 1. The concentration of B-END-like immunoreactivity per $\mathrm{mg}$ protein for caudal medulla oblongata and hypothalamus. Results are expressed as the mean + S.E.M. for 5 animals per time point. The inset demonstrates alterations in the levels of the B-END-like immunoreactivity for embryonic day 21 and postnatal day 1 . The units are expressed as concentration in femtomoles of immunoreactivity per mg protein. CMO, caudal medulla oblongata; HYPO, hypothalamus; E21, embryonic day 21; P1, postnatal day 1; A, adult; $\mathrm{B}$-END, $\beta$-endorphin.

hour, with $1.1 \mathrm{ml}$ per fraction. The collected samples were lyophilized and stored at $-80^{\circ} \mathrm{C}$. At the time of the assay, the samples were resuspended in $1 \mathrm{ml}$ of $1 \%$ formic acid. The number of regions pooled for the sample for embryonic day 21 was 55 for the caudal medulla oblongata and 60 for the hypothalamus. The number of regions pooled for the hypothalamus and for the caudal medulla oblongata for postnatal day 1 was 65 and the adult was 15 . The identification of the immunoreactive peaks was performed by comparison with known standards using B-LPH which was donated by Dr. C.H. Li (Department of Psychiatry at the University of California San Francisco) and B-END (1-3i), (1-27), (1-26) (Peninsula Laboratories). The POMC peak was determined by identifying the immunoreactive peak with the shortest retention time since this peptide is the highest molecular weight peptide that is immunoreactive to the B-END radioimmunoassay.

\section{Statistical analysis}

The statistical analysis on the individual timepoint B-END levels of immunoreactivity was performed using single factor ANOVA and post-hoc analysis. The level of significance was $\alpha=0.05$. The ratios of immunoreactive peptides were calculated using the summed immunoreactivities of the fractions.

\section{RESULTS}

\section{B-END levels}

The values (mean \pm S.E.M.) for B-END-like immunoreactivity per milligram protein in the caudal medulla oblongata was $14.1 \pm 3.0 \mathrm{fmol} / \mathrm{mg}$ protein for embryonic day $21,12.9 \pm 5.4 \mathrm{fmol} / \mathrm{mg}$ protein for postnatal day 1 and $141.5 \pm 6.1 \mathrm{fmol} / \mathrm{mg}$ protein for the adult. There was no statistical difference between embryonic day 21 and postnatal day 1; however, postnatal day 1 was statistically different from the adult $(P<0.003)$ as was embryonic day 21 from adult $(P<0.04)$.

The values for the concentration of B-END in the hypothalamus as depicted in Fig. 1 were $17.5 \pm 1.6 \mathrm{fmol} / \mathrm{mg}$ protein for embryonic day $21,21.6 \pm 3.6 \mathrm{fmol} / \mathrm{mg}$ pro- 
tein for postnatal day 1 and $768.5 \pm 50.9 \mathrm{fmol} / \mathrm{mg}$ protein for the adult. There was no statistical difference between embryonic day 21 and postnatal day 1 for the hypothalamus; however, there was a statistical difference between embryonic day 21 and adult $(P<0.01)$ as well as postnatal day 1 and adult $(P<0.02)$.

\section{Chromatography}

Fig. 2 represents the patterns of B-END-like immu- noreactive material separated by molecular exclusion chromatography for ages embryonic day 21, postnatal day 1 and adult. Percentages of peak immunoreactivity for the peptides were calculated based on total immunoreactivity found by radioimmunoassay of the chromatographic fractions. The total sum of immunoreactive peaks found during chromatographic separation of the pooled caudal medulla oblongata regions were 599.5 fmol for embryonic day $21,1452.2$ fmol for postnatal day
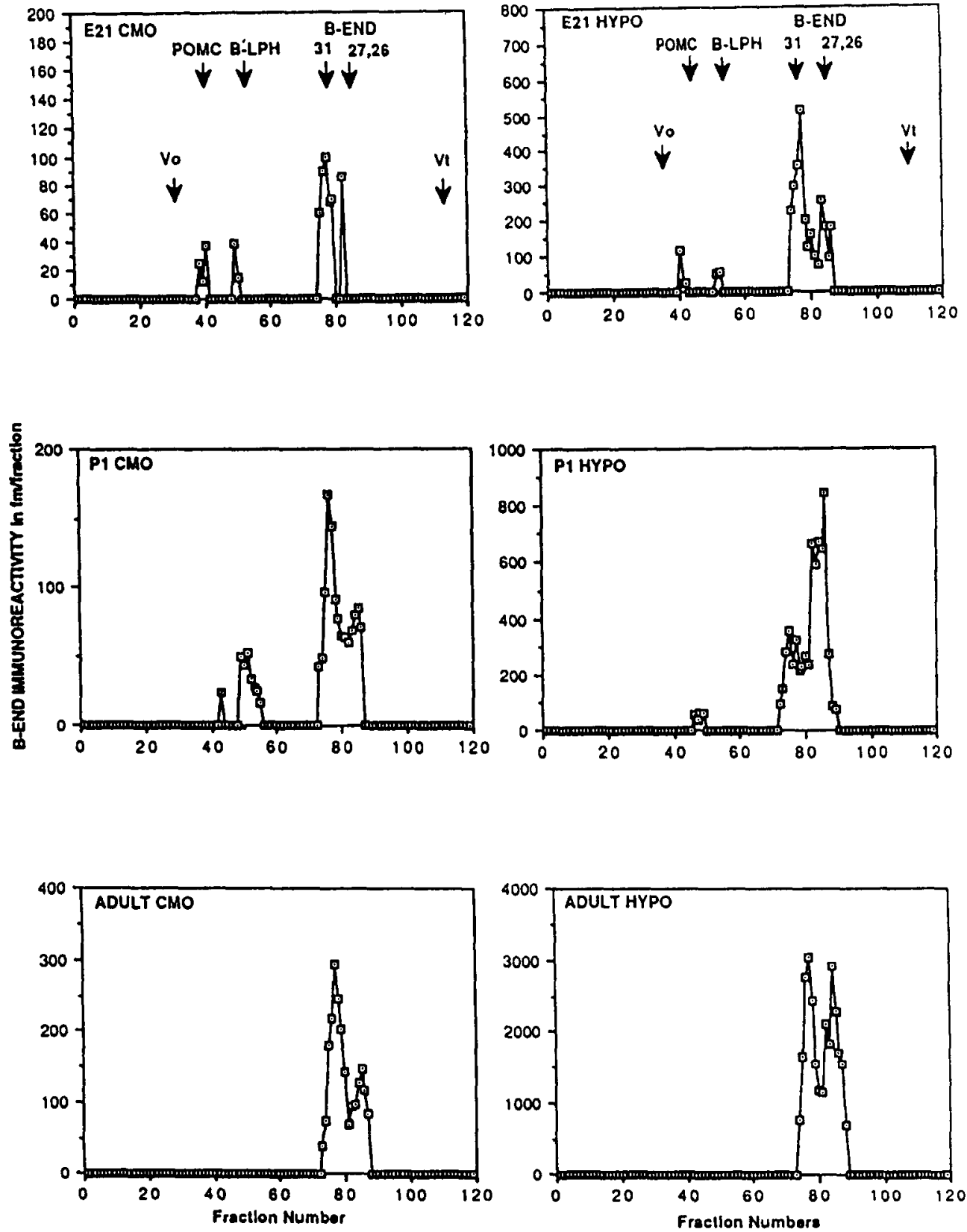

Fig. 2. The chromatographic patterns of B-END-like immunoreactive material for the caudal medulla oblongata and hypothalamus for individual time point pooled tissue samples from embryonic day 21, postnatal day 1 and adult. Aliquots from extracts of 55 embryonic days 21 nucleus tractus solitarii, 60 embryonic day 21 hypothalamus, 65 postnatal day 1 for nucleus tractus solitarii and hypothalamus and 15 adult nucleus tractus solitarii and hypothalamus were separated on Sephadex G50-50 superfine $15 \times 900 \mathrm{~mm}$ column with $1.1 \mathrm{ml}$ per fraction and assayed for B-END. For further details see Material and Methods. Void volume was determined with bovine serum albumin and total volume with 2-mercaptoethanol. 


\section{TABLE I}

The ratios of the immunoreactivity calculated for the B-END 31 residue and the 27,26-residue peptides in the hypothalamus and caudal medulla oblongata at embryonic day 21 , postnatal day $I$ and adult

The chromatographic peak of the 27-residue B-END peptide overlapped the 26-residue peptide peak, so the immunoreactivities were combined to calculate the ratios.

\begin{tabular}{|c|c|c|c|c|}
\hline \multirow[t]{2}{*}{ Age } & \multicolumn{2}{|l|}{ Medulla } & \multicolumn{2}{|c|}{ Hypothalamus } \\
\hline & $\begin{array}{l}B-E N D \\
(1-31)\end{array}$ & $\begin{array}{l}B-E N D \\
(1-27,26)\end{array}$ & $\begin{array}{l}B-E N D \\
(I-3 I)\end{array}$ & $\begin{array}{l}B-E N D \\
(1-27,26)\end{array}$ \\
\hline Embryonic day 21 & 4.48 & 1 & 2.11 & 1 \\
\hline Postnatal day 1 & 1.79 & 1 & 1 & 1.90 \\
\hline Adult & 2.19 & 1 & 1 & 1.06 \\
\hline
\end{tabular}

1 and $2154.5 \mathrm{fmol}$ for adult tissue pools. For the caudal medulla oblongata tissue, the percentage of POMC immunoreactivity decreased across the 3 time points with a value of $12.4 \%$ ( $74.5 \mathrm{fmol} / \mathrm{peak})$ for embryonic day 21 to $1.6 \%(23.3 \mathrm{fmol} / \mathrm{peak})$ for postnatal day 1 . No immunoreactivity could be assayed in the fractions in the vicinity of the expected POMC peak for the adult tissue. The percentage of B-LPH rose from 9.0\% $(54.0 \mathrm{fmol} /$ peak) for embryonic day 21 to $15.6 \%$ (226.1 fmol/peak) for postnatal day 1. B-LPH could not be detected in the vicinity of similar fractions for the adult tissue. The percentage of the total B-END immunoreactivity, i.e. both the 31- and 27,26-residue peptides was $78.6 \%$ (471.0 fmol/peak) for embryonic day $21,82.8 \%$ (1202.8 fmol/ peak) for postnatal day 1 and $100 \%$ (2154.8 fmol/peak) for the adult.

The total immunoreactivity for the sum of the peaks measured in the pooled hypothalamic chromatographic samples was $3036.7 \mathrm{fmol}$ for embryonic day $21,6454.4$ fmol for postnatal day 21, and 27,764.9 fmol for the adult. The hypothalamic POMC peak percentages decreased more rapidly than the nucleus tractus solitarii with embryonic day 21 at $5.5 \%$ ( $165.6 \mathrm{fmol} /$ peak) and $0 \%$ for both postnatal day 1 and adult. The percentage of hypothalamic B-LPH for embryonic day 21 was $3.5 \%$ (105.6 fmol/peak), postnatal day 1 was $3.4 \%(217.8 \mathrm{fmol} /$ peak) and $0 \%$ in the adult. The peak percentage for the total B-END in the hypothalamus was $91.0 \%$ (2765.6 fmol/peak) at embryonic day $21,96.6 \%(6236.7 \mathrm{fmol} /$ peak) for postnatal day 1 and $100 \%$ for the adult.

\section{DISCUSSION}

The results indicate that the relative concentration of B-END-like immunoreactivity does not fluctuate significantly between embryonic day 21 and postnatal day 1 in the caudal medulla oblongata and the hypothalamus.
An alteration did occur in the post-translational processing of the POMC and related peptides, demonstrated by a shift in the pattern of processing from a high molecular weight precursor to intermediate and low molecular weight peptides for both the caudal medulla oblongata and the hypothalamus between embryonic day 21 and postnatal day 1 . The difference in the rate of post-translational processing of POMC between the caudal medulla oblongata and the hypothalamus may occur either as a result of an immature enzymatic proteolysis system or immunoreactive material originating from another source. The caudal medulla oblongata may not contain as high a concentration of proteolytic enzymes or the enzymes may not be activated to the same extent as found in the hypothalamus. The potential for material originating from other sites has been demonstrated by Alessi and Quinlan ${ }^{4}$, Van der Kooy ${ }^{28}$ and Gray ${ }^{10}$. Alessi demonstrated in monosodium glutamate (MSG)-treated rats that there was a significant drop in the concentration of B-END-like immunoreactivity in the ventral section of the caudal medulla oblongata while the dorsal section did not statistically differ from saline control animals. No immunocytochemical changes were found in the nucleus tractus solitarii between the saline and MSGtreated animals. The existence of a route for transport of peptides into the caudal medulla oblongata was demonstrated by Van der Kooy. In this study, retrograde track tracing revealed projections from cell bodies in the hypothalamus to the medulla oblongata ${ }^{22}$. Gray, utilizing combined fluorescent retrograde tracer and immunofluorescence techniques, located axons from POMC cells in the lateral arcuate regions of the hypothalamus extending to the nucleus tractus solitarii-dorsal vagal complex ${ }^{10}$. In our study the caudal medulla oblongata was not subdivided into ventral and dorsal sections and POMC peptides in the tissue may have originated from another site.

The cleavage of the 31-residue B-END peptide differed between the caudal medulla oblongata and hypothalamus. For the caudal medulla oblongata, the decrease in the ratio of the immunoreactivity of 31-residue peak to the 27,26-residue peak between embryonic day 21 and postnatal 1 may be due to either an increase in proteolytic enzyme activity or an increased concentration of the immunoreactive material originating from perikarya outside the dissected region. The hypothalamus had a more dramatic shift in the ratios between embryonic day 21 and postnatal day 1. The 31-residue peptide immunoreactivity predominated at greater than a 2 to 1 ratio at embryonic day 21 while at postnatal day 1 the 27,26-residue immunoreactivity predominated at a ratio nearly 2 to 1 . Smyth ${ }^{27}$ and Bleakman ${ }^{5}$ have demonstrated that proteolytic processing of POMC occurs in 
a sequential fashion, therefore no C-terminal proteolytic processing occurs on the B-LPH peptide. The formation of the 27, and 26-residue B-END peptides is from the 31-residue peptide. The dramatic ratio shifts in the hypothalamus appear to be activation of the C-terminal proteolysis system occurring at the time of parturition. In the case of the caudal medulla oblongata, the ratio simply decreases between the 31-residue and 27,26-residue peptides.

In conclusion, the tissue levels and concentration of B-END are relatively consistent prior to and following

\section{REFERENCES}

1 Allen, R.G., Pintar, J.E., Stack, J. and Kendall, J.W., Biosynthesis and processing of pro-opiomelanocortin-derived peptides during fetal pituitary development, Dev. Biol., 102 (1984) 4350.

2 Alessi, N.E. and Khachaturian, H., Postnatal development of beta-endorphin immunoreactivity in the medulla oblongata of rat, Neuropeptides, 5 (1985) 473-476.

3 Alessi, N.E and Quinlan, P., Postnatal development of ACTH and alpha-MSH in the medulla oblongata of rat: alpha-MSH is the predominant peptide, Peptides, 6 (1985) 137-141.

4 Alessi, N.E., Quinlan, P. and Khachaturian, H., MSG effects of beta-endorphin and alpha-MSH in the hypothalamus and caudal medulla, Peptides, 9 (1988) 689-695.

5 Bleakman, A., Bradbury, A.F., Darby, N.J., Maruthainur, K. and Smyth, D.G., Processing reactions in the later stages of hormone action, Biochimie, 70 (1988) 3-10.

6 Cahill, C.A., Matthews, J.D. and Akil, H., Human plasma beta-endorphin-like peptides: a rapid high recovery extraction technique and validation of radioimmunoassay, J. Clin. Endocrinol. Metabol., 56 (1983) 992-997.

7 Deakins, J.W.F., Dostrovsky, J.O., Smyth, D.B., Influence of N-terminal acetylation and C-terminal proteolysis on analgesic activity of beta-endorphin, Biochem. J., 189 (1980) 501-506.

8 Eipper, B.A. and Mains, R.E., Extistence of a common precursor to ACTH and endorphin in the anterior and intermediate lobes of rat pituitary, J. Supramol. Struct., 8 (1978) 247256.

9 Glowinski, J. and Iverson, L.L., Regional studies of catecholamines in the rat brain. The disposition of $\left[{ }^{3} \mathrm{H}\right]$ norepinephrine $\left[{ }^{3} \mathrm{H}\right]$ dopamine, and $\left[{ }^{3} \mathrm{H}\right]$ DOPA in various regions of the brain, J. Neurochem., 13 (1966) 655-669.

10 Gray, T.S., O'Donohue, T.L., Watson, S.J. and Magnuson, D.J., Pro-opiomelanocortin and neuropeptide $Y$ projections from the arcuate and periarcuate hypothalamic areas to the nucleus tractus solitarius-dorsal vagal complex, Soc. Neurosci. Abstr., 10 (1984) 432.

11 Hazinski, T.A., Schlueter,M.A., Tooley, W.H. and Grunstein, M.M., Effect of naloxone on ventilation in unanesthetized newborn rabbits, J. Appl. Physiol., 50 (1981) 713-717.

12 Khachaturian, H., Alessi, N.E., Munfakh, N., Watson, S.J., Ontogeny of opioid and related peptides in the rat CNS and pituitary: an immunocytochemical study, Life Sci., 33 (1983) 6164.

13 Khachaturian, H., Lewis, M.E., Alessi, N.E. and Watson, S.J., Time of origin of opioid peptide-containing neurons in the rat hypothalamus, J.Comp. Neurol., 236 (1985) 538-546.

14 Kreiger, D.T., Liotta, A.S., Brownstein, M.J., Zimmerman, E.A., ACTH, $\beta$-lipotropin and related peptides in brain, pituitary, and blood, Recent Prog. Horm. Res., 36 (1980) 277-286.

15 Lakke, E.A.J.F. and Marani, E., A zonal pattern of neuroglial parturition in the caudal medulla oblongata and the hypothalamus. The occurrence of parturition in the rat, however, is associated with alterations in peptide forms within the fetus and neonate. The changes are not limited to one site in the brain, thus influences on neuronal processes occur at multiple levels. Thus if B-END is to be implicated in conditions such as Sudden Infant Death Syndrome, the mechanism may go beyond absolute tissue levels of the peptide in the brainstem to include alterations in proteolysis, as well as extramedullary sources of the B-END-like peptides.

cells during the development of the intermediate lobe of the rat pituitary, Neurosci. Lett., 93 (1988) 146-151.

16 Lowry, O.H., Rosebrough, N.Y, Farr, A.L. and Randall, R.J., Protein measurement with Folin phenol reagent, J. Biol. Chem., 193 (1951) 265-275.

17 Main, R.E. and Eipper, B.A., Common precursor to corticotropins and endorphins, Proc. Natl. Acad. Sci. U.S.A., 74 (1977) 3014.

18 Marani, E. and Van der Veeken, J.G., The postnatal developmental localization of pro-opiomelanocortin and alpha-melanocyte stimulating hormone in the medio-basal hypothalamus of the rat, Basic Appl. Histochem., 32 (1988) 171-178.

19 Moss, I.R. and Scarpelli, E.M., Beta-endorphin central depression of respiration and circulation, J. App. Physiol., 50 (1981) 1011-1016.

20 Nicolas, P., Hammonds, R.G. and Li, C.H., Beta-endorphininduced analgesia is inhibited by synthetic analogs of beta-endorphin, Proc. Natl. Acad. Sci. U.S.A., 81 (1984) 3074- 3074.

21 Pasi, A., Foletta, D., Molz, G., Hartmann, H., Gramsch, C., Mehraein, P. and Pedrinis, E., Regional levels of beta-lipotropin and beta-endorphin in the brain and hypophysis of sudden infant death syndrome victims, Arch. Pathol. Lab. Med., 107 (1983) 336-337.

22 Pasi, A.P., Kulling, D., Voellmy, C., Gramsh, P., Mehraein, M., Hani and Messiha, F.S., Beta-endorphin in the brainstem and the cerebellum of the human infant: regional levels' profile assessed with immunoaffinity chromatography and solid phase radioimmunoassay, Physiol. and Behav., 46 (1989) 13-16.

23 Schwartzberg, D.G. and Nakane, P., Ontogenesis of adrenocorticotropin-related peptide determinants in the hypothalamus and pituitary gland of the rat, Endocrinology, 110 (1982) 855-864.

24 Scott, A.P., Lowry, P.J., Ratcliffe, J.G., Rees, L.H., Landon, J., Corticotropin-like peptides in the rat pituitary, J. Endocrinol., 61 (1974) 355-361.

25 Sato, S.M. and Mains, R.E., Post-translational processing of proadrenocorticotropin/endorphin-derived peptides during postnatal development in the rat pituitary, Endocrinology, 117 (1985) 773-786.

26 Seizenger, B.R., Hollt, V. and Herz, A., Postnatal development of $\beta$-endorphin-related peptides in rat anterior and intermediate lobes: evidence for contrasting development of proopiomelanocortin processing, Endocrinology, 115 (1984) 136-142.

27 Smyth, D.G., Darby, N.J. and Maruthainar, K., Sequential formation of beta-endorphin-related peptides in the porcine pituitary, Neuroendocrinology, 47 (1988) 317-322.

28 Van der Kooy, D., Koda, L.Y., McGinty, J.F., Gerfen, C.R. and Bloom, F.E., The organization of projections from the cortex, amygdala, hypothalamus to the nucleus of solitary tract in rat, J. Comp. Neurol., 224 (1984) 1-24.

29 Yeung, H.W., Yamashiro, D., Chang, W.C. and Li, C.H., Synthesis and opiate activity beta-endorphin analogs with various chain lengths, Int. J. Pept. Protein Res., 12 (1978) 42-48. 